\title{
Research Paper: Effect of Acceptance and Commitment Group Therapy on Social Ad- justment and Social Phobia Among Physically-Disabled Persons
}

\author{
Zahra Ostadian Khani ${ }^{1}$, *Maliheh Fadaie Moghadam²
}

1. Department of General Psychology, Faculty of Psychology, Central Tehran Branch, Islamic Azad University, Tehran, Iran. 2. Department of Clinical Psychology, Faculty of Psychology, South Tehran Branch, Payame Noor University, Tehran, Iran.

Citation: Ostadian Khani Z, Fadaie Moghadam M. [Effect of Acceptance and Commitment Group Therapy on Social Adjustment and Social Phobia Among Physically-Disabled Persons (Persian)]. Archives of Rehabilitation. 2017; 18(1):63-72. https://doi.org/10.21859/jrehab-180163

: https://doi.org/10.21859/jrehab-180163

Received: 12 Sep. 2016

Accepted: 06 Dec. 2016
Keywords:

Acceptance and commitment group therapy, Social adjustment, Body image, Social phobia

\section{ABSTRACT}

Objective Human behavior is a function of one's existence and interaction between body and mind. Disability is the inability to perform all or part of the normal activities of individual or social life due to congenital defects, or physical or mental faculties being subject to accidents. Mental disability rehabilitation approach consists of three categories of services: mental, emotional, and physical. Physical or motor disabilities are divided into four categories: physical, visceral, sensory and aging. The concept of disability is omnipresent in every society and culture. Its form may vary between temporary or permanent, total or partial, and fixed or variable. Side effects of disability on individuals are different according to their attitude. Their views about themselves, disability, and familial and social attitudes leads to various complications in the lives of persons with disabilities. It has been proved that endangering the physical health provides psychological reactions in people. Many physical disabilities are associated with mental health problems. Mental health problems in people with health problems are very common. For example, chronic diseases, create physical conditions and limiting long-term negative consequences for the disabled person. Quasi-physical as well as psychological problems have serious implications with respect to diseases. Psychosomatic diseases, for instance, cause different kinds of disabilities. In addition to the development of the machines, the marriage and many other issues may be associated with increased physical disability and mental illness. The importance of physical disabilities due to mental health problems that are associated with health problems treated, including psychiatric problems that disabled people face; social is the low and high social phobia. The aim of this study was to determine the effectiveness of acceptance and commitment group therapy based on social adjustment and social phobia among physically-disabled person.

Methods \& Materials The study population consisted of all individuals belonging to a disability welfare organization in Qom. The sample included 30 males (15 in the control group and 15 in the experimental group) who were selected by random sampling method. In this quasi-experimental study, the participants in the experimental group underwent a 2-month intervention of eight 120-minute sessions. The research design was pretest-posttest with a control group. Data were collected from the questionnaires of Conover, social adjustment, Weisman, and social phobia. Analysis of covariance (ANCOVA) model was used for analyzing. Results Our results showed that the acceptance and commitment group therapy increased satisfaction as far as social adjustment was concerned $(P<0.001)$. Significant effect on the subscales of fear $(P<0.001)$, avoidance $(P<0.001)$, physiology $(P<0.001)$, and the total social phobia score $(P<0.001)$ was also observed.

Conclusion The acceptance and commitment group therapy increased social adjustment and decreased social phobia among physically-disabled persons.

\section{* Corresponding Author:}

Zahra Ostadian Khani, PhD Candidate

Address: Department of Clinical Psychology, Faculty of Psychology, Central Tehran Branch, Islamic Azad University, Tehran, Iran.

Tel: +98 (912) 8517245

E-Mail: m.2001.moghadam@gmail.com 


\title{
اثربخشى تروهدرمانى مبتنى بر يذيرش و تعهدبر ساز گارى اجتماعى وهراس اجتماعى معلولان جسمى
}

\author{
زهرا استاديان خانى'، "مليحه فدايى مقدم'
}

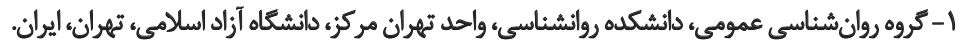

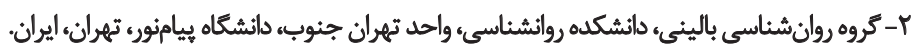

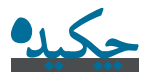

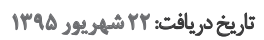

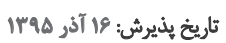

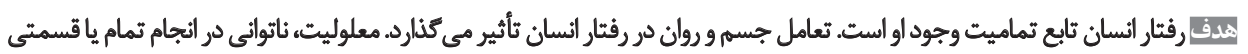

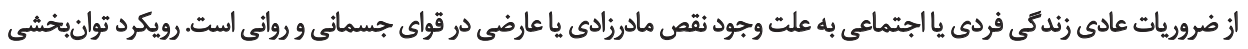

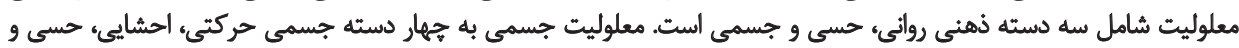

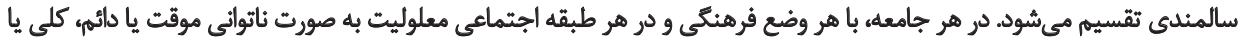

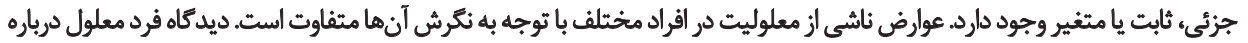

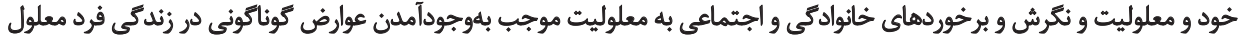

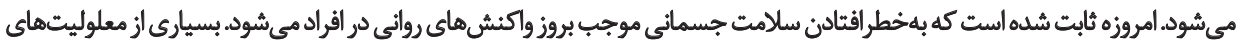

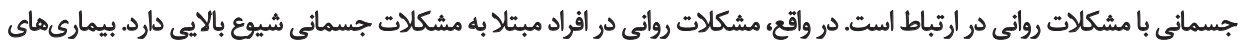

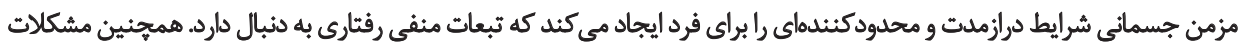

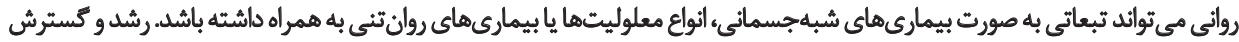

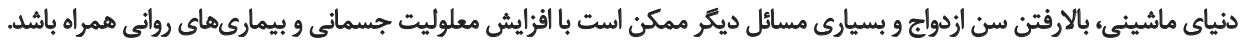

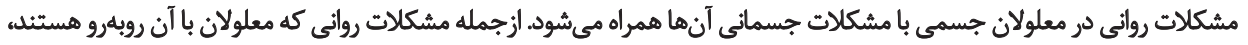

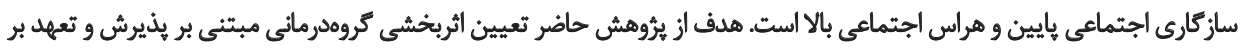

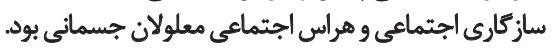

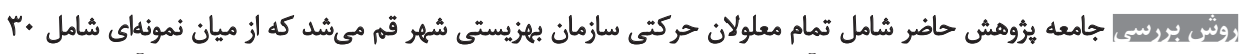

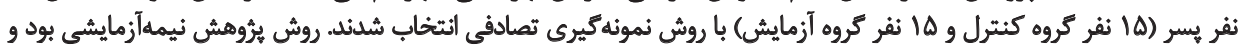

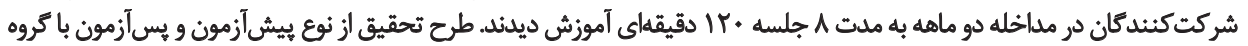

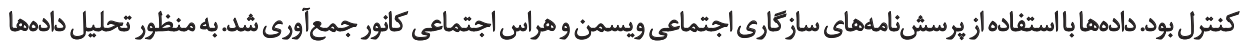

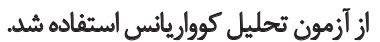

كليدوازٔهها:

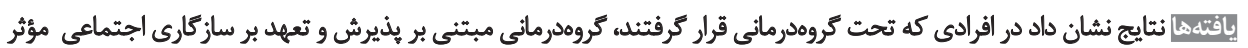

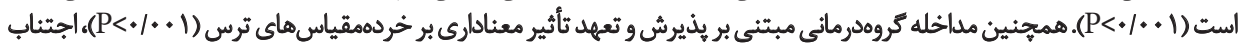

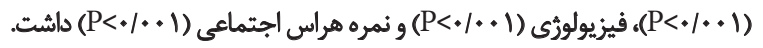

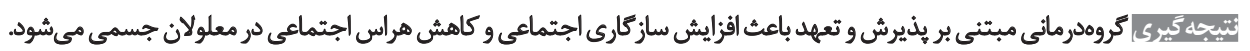

كروه درماني مبتني

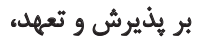
سازكارى اجتماعى ونيء هراس اجتماعي اجتماعي

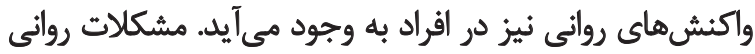

مقدمه

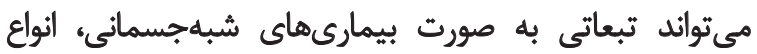

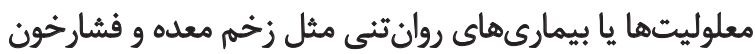

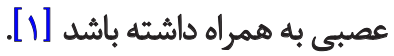

معلوليت عبارت است از ناتوانى در انجام تمام يا قسمتى از

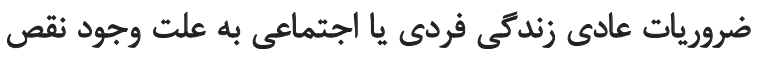

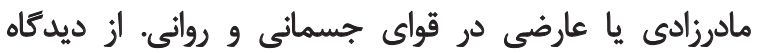

وقتى انسان از نظر جسمى سالم باشد يرانرزى ترتر، شادتر،

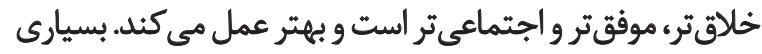

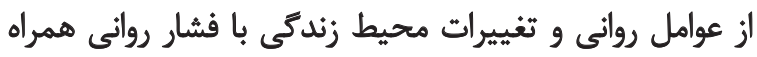

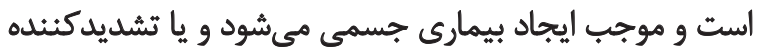
تظاهرات اين بيمارىهاست. با بهخطرافتادن سلامت جسماني، 


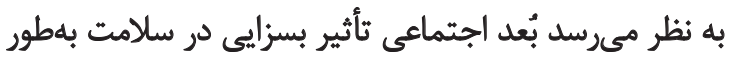

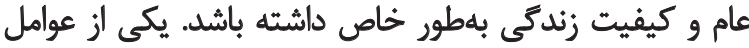

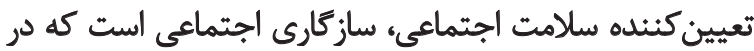

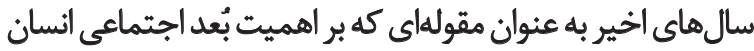

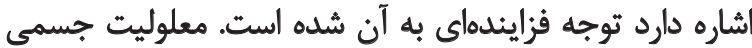

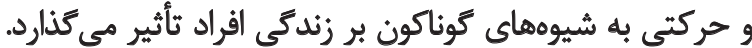

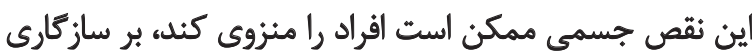

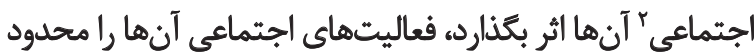

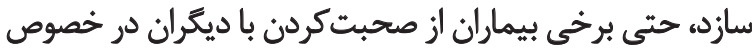

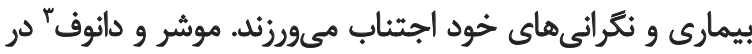

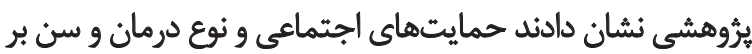

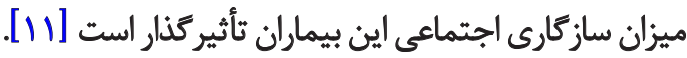

طبق نظر اريندل"، سازخًارى اجتماعى ناراحتى و ترسى است

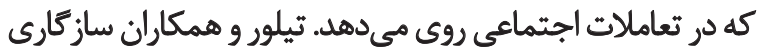

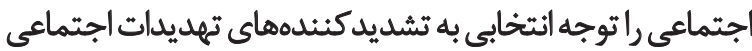

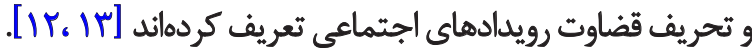

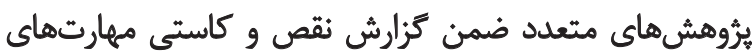

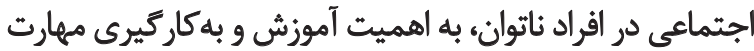

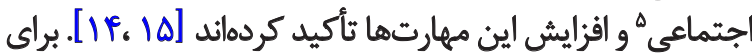

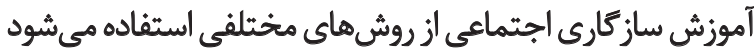

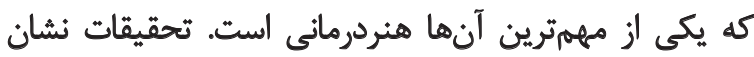

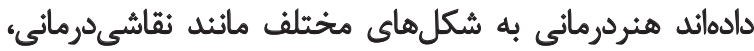

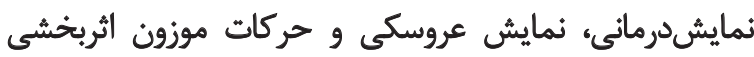

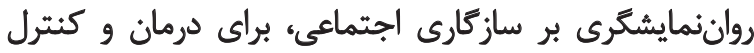

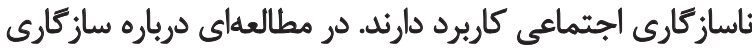

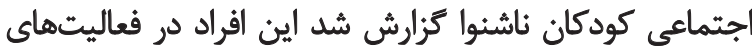

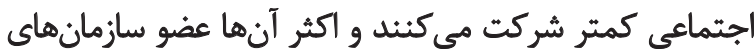

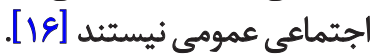

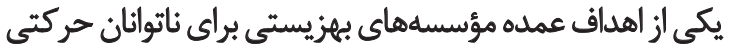

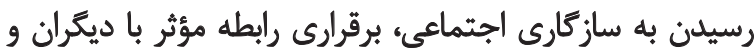

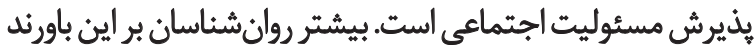

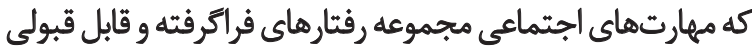

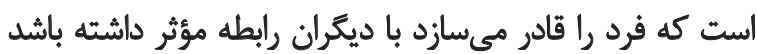

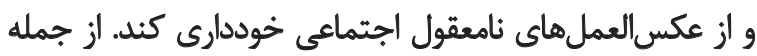

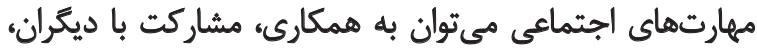

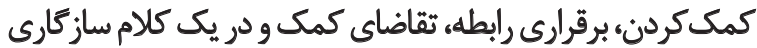

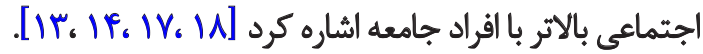
يرؤششها مؤيد اين نكته است كه سازكارى اجتماعى يايين

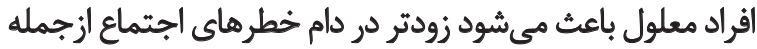

2. Social adjusment

3. Mosher \& Danoff

4. Arrindell

5. Social Skills
توانبخشى معلوليتها به سه دسثه ذهنى روانى، حسى و

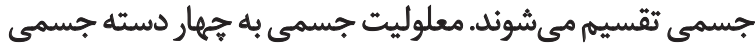

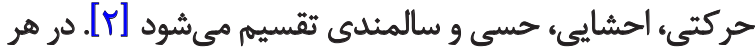

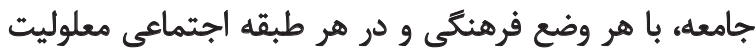

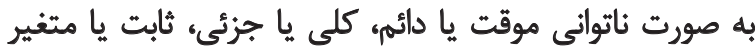

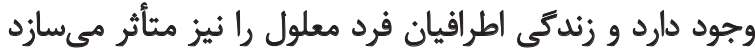

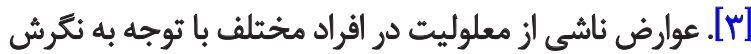

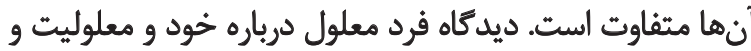

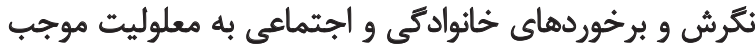

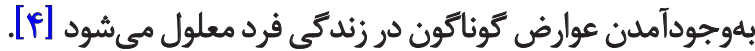
يكى از شايعترين اختلالات مزمن ناشى از عوارض معلوليت،

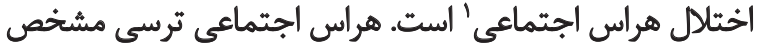

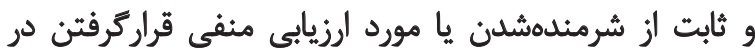

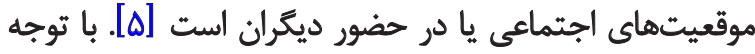

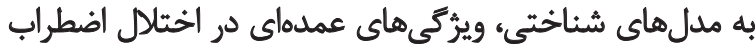

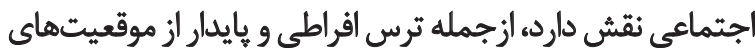

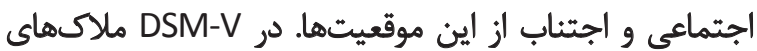

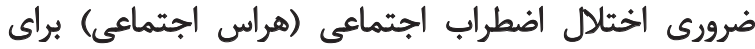

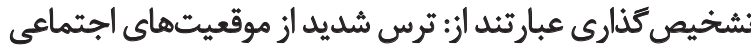

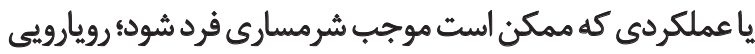

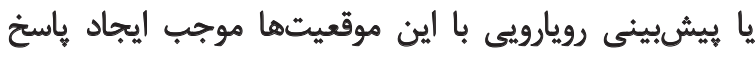

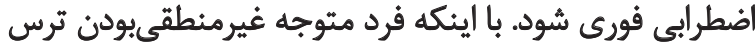

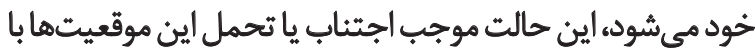

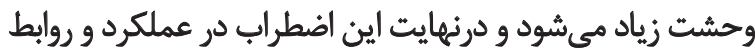

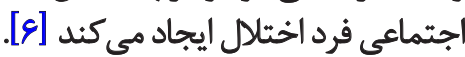

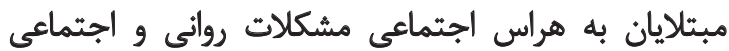

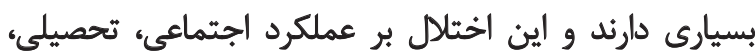

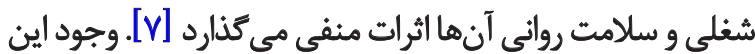

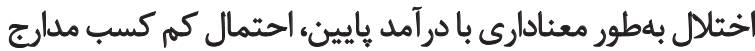

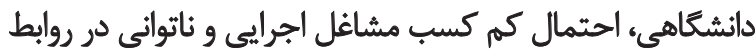

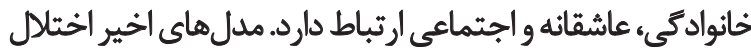

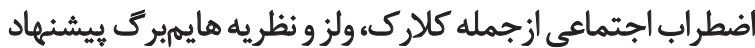

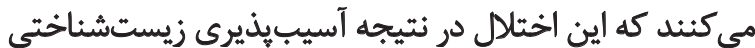

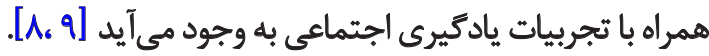
جا توجه به اينكه معلوليت در ابعاد مختلف اجتماعي و اقتصادى

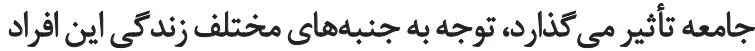

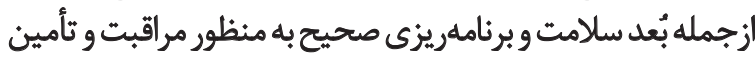

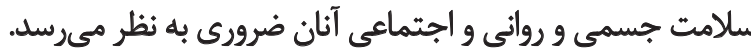

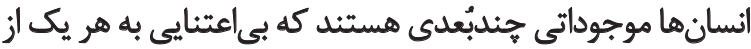

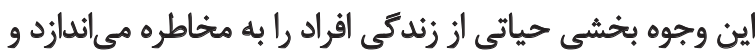

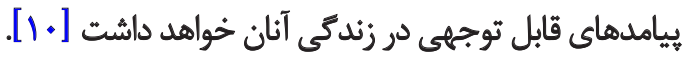


و درونى يذيرفت. در مرحله بعد به فرد آموخته ميىشود كه از إز

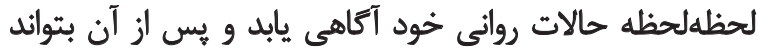

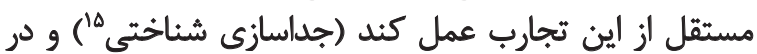

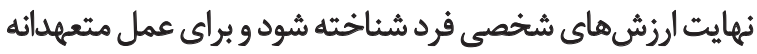

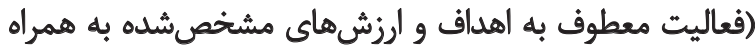
يذيرش تجارب ذهنى) انكيزه ايجاد شود.

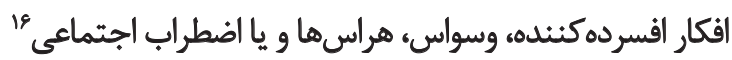

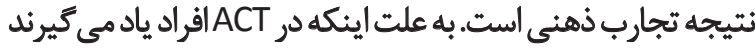

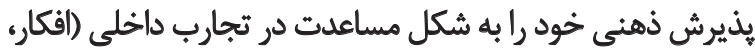

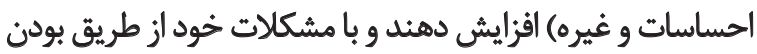

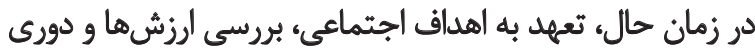

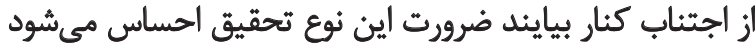

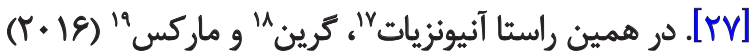

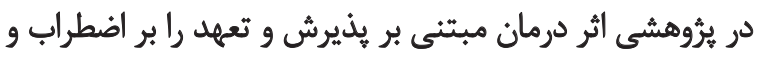

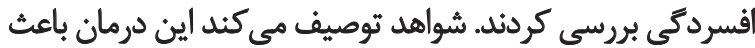

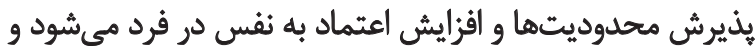

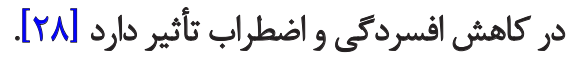

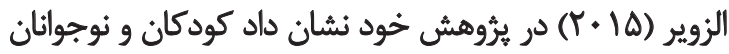

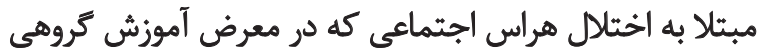

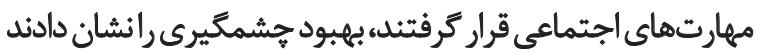

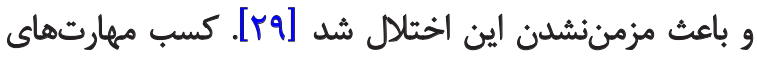

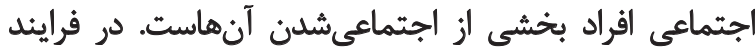

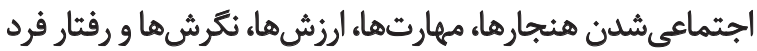

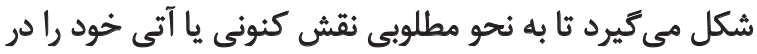

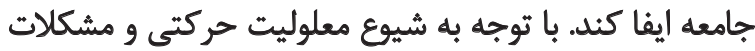

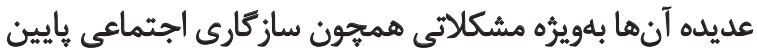

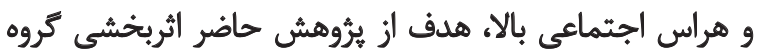

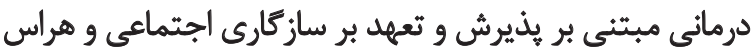

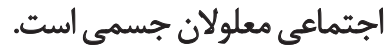

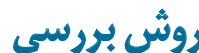

يُروهش حاضر از نظر هدف، آيندهنكّر و بنيادى كاربردى، از نظر

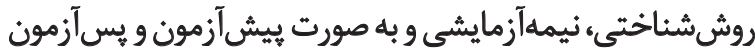

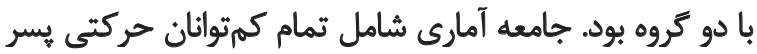

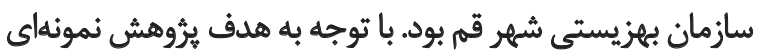

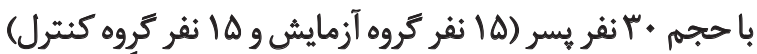

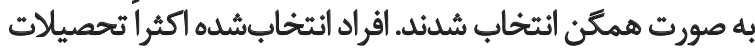

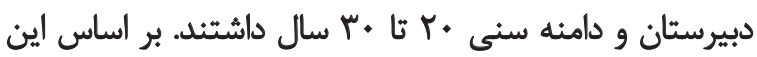

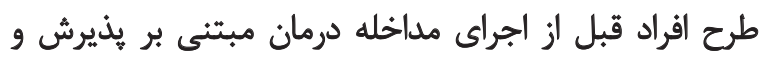

15. Cognitive isolated

16. Social anxiety

17. Annunziaj

18. Green

19. Marx

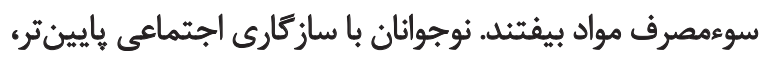

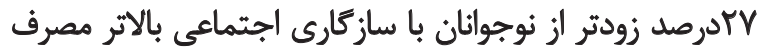

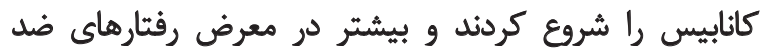

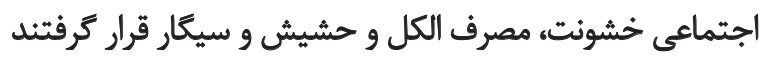

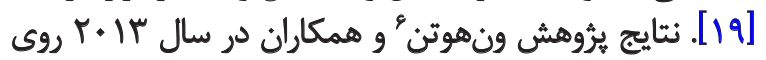

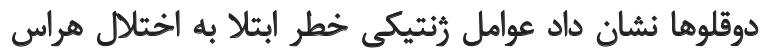

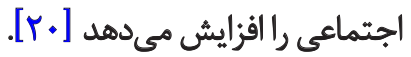

متخصصان بهداشت روانى علاوه بر درمان دارويى براى درمان اندئ

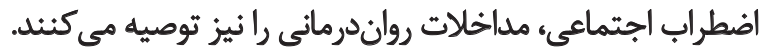

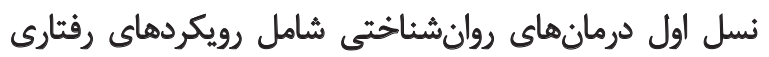

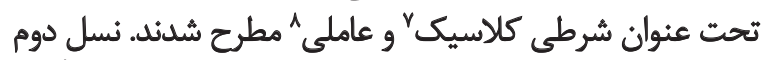

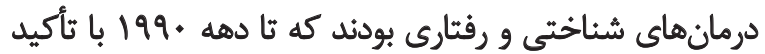

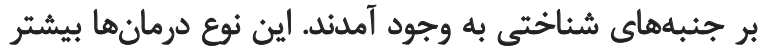

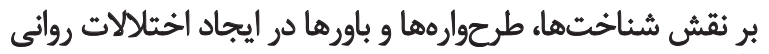

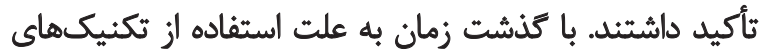

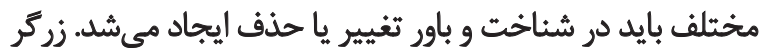

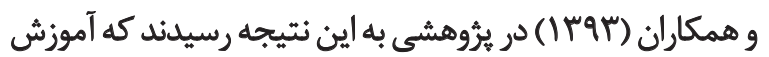

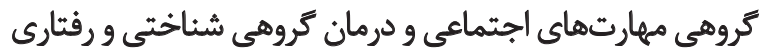

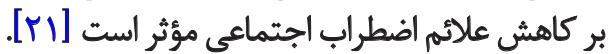

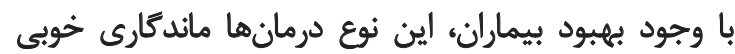

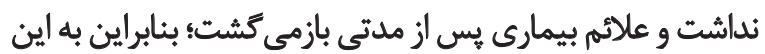

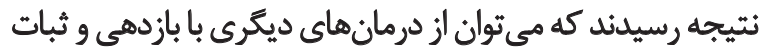

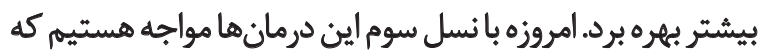

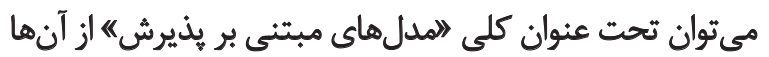

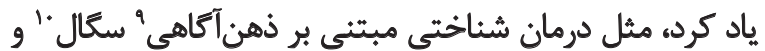

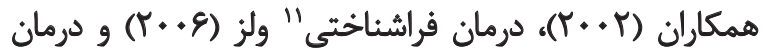

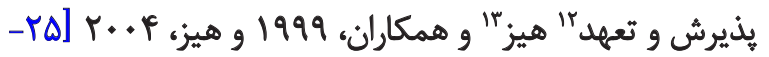

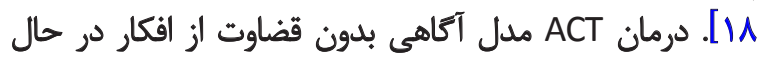

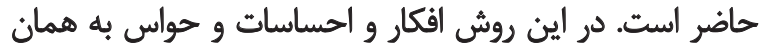

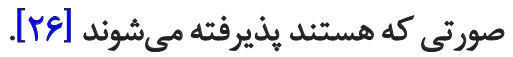

در اين درمانها به جاى تغيير شناختها سعى مي إشود تأنا

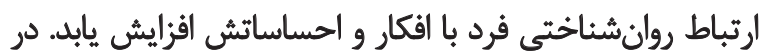

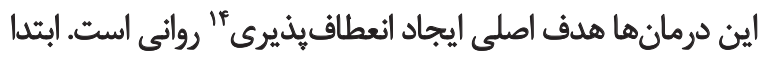

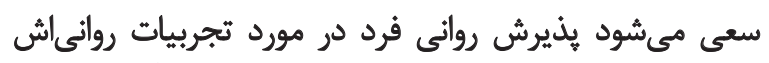

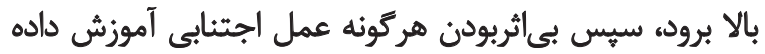

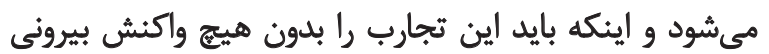

6. Van Houten

7. Classical conditioning

8. Conditioning factor

9. Mindfulness

10. Segal

11. MCT

12. ACT

13. Hayes

14. Flexibility 
درمان داروييى و رواندرمانى بيماران افسرده طراحى شده است. در

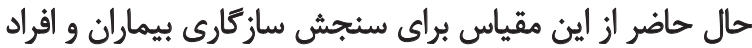

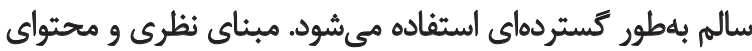

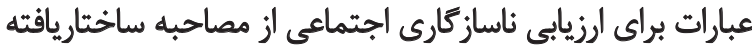

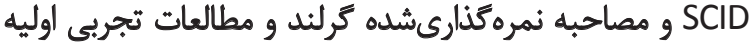

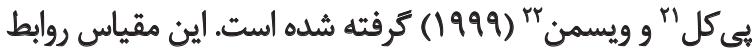

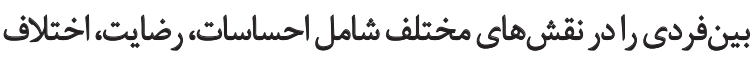

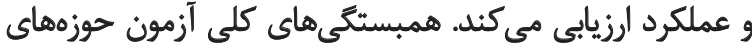

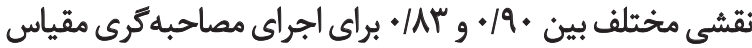

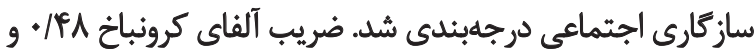

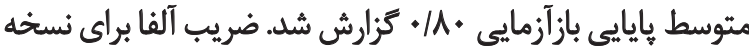

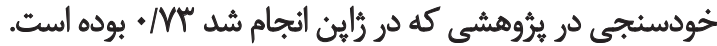

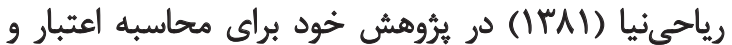

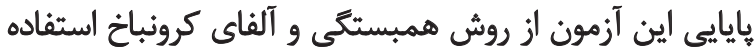

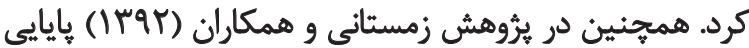

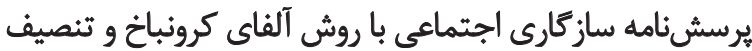

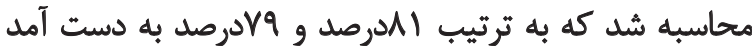

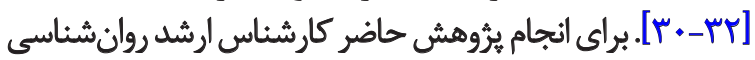

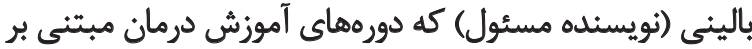
بذيرش و تعهد را كذارنده است كروهدرمانى را انجام داد. شرح دران

21. Paykol

22. Wissman

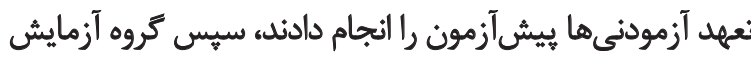

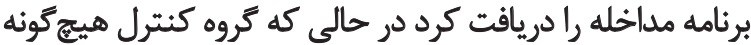

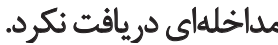

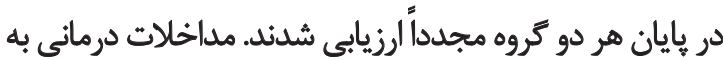

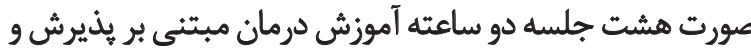

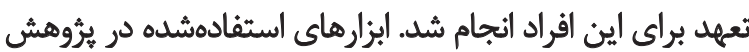

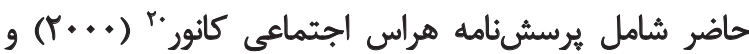
يرسشنامه سازگًارى اجتماعى يى كل و ويسمن (1999) بود.

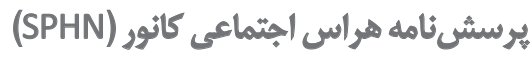

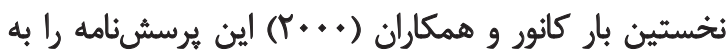

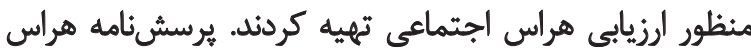

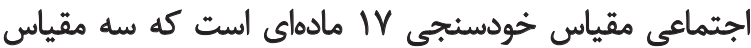

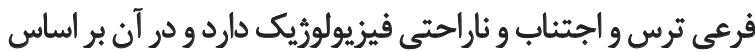

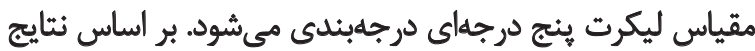

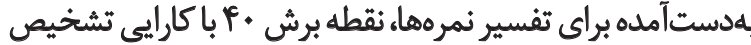

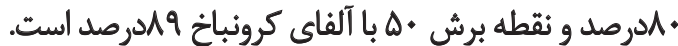

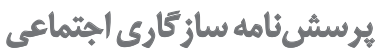

مقياس ساز كارى اجتماعى به عنوان مقياس برآيند براى ارزيابى

20. Kanour

جدولا. شرح جلسات تروه درمانى مبتنى بر بذيرش و تعهد

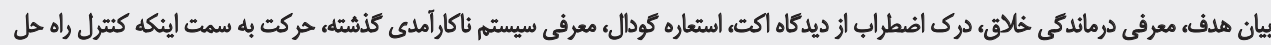

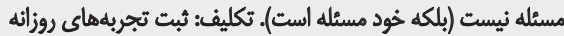
جلسه اول

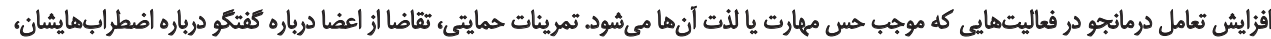

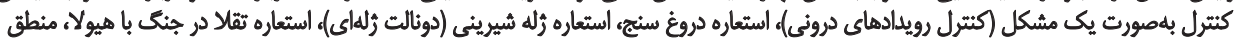

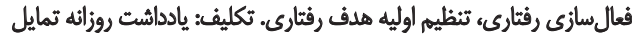

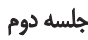

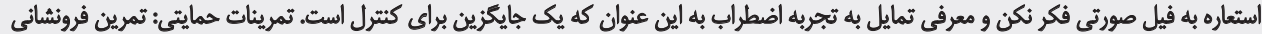

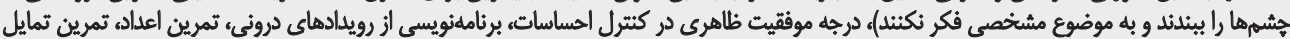

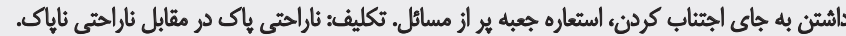

:

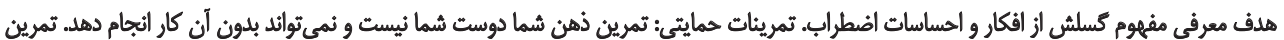

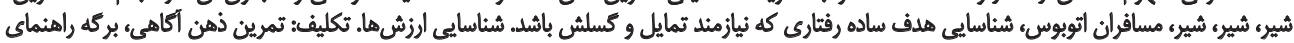

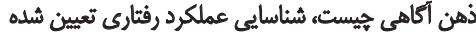

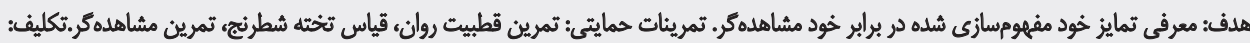

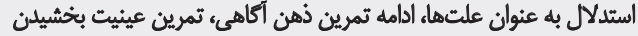

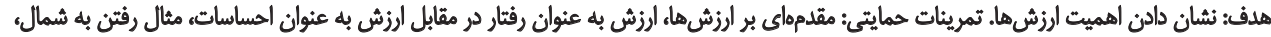

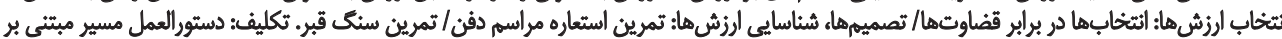

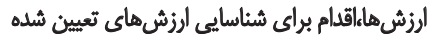

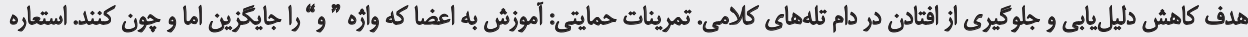

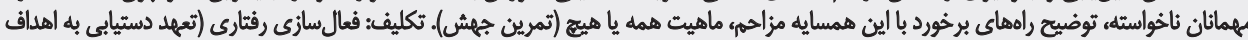
كوجكتر تعيين شلهة). 


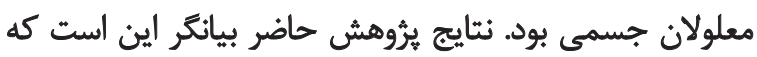

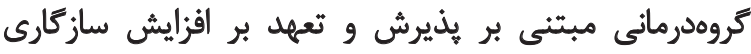

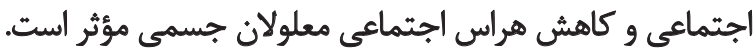

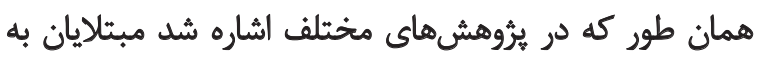

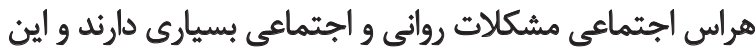

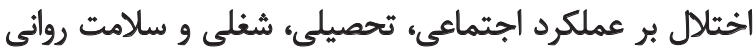

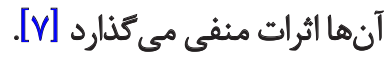

وجود اين اختلال بهطور معنادارى با درآمد بإيين، احتمال

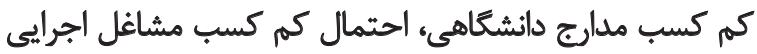

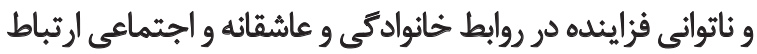

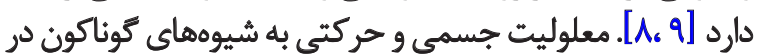

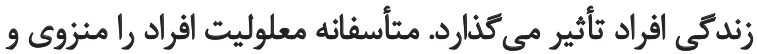

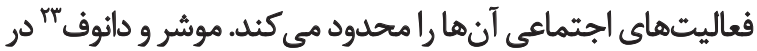

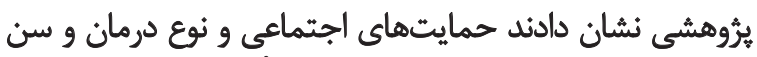

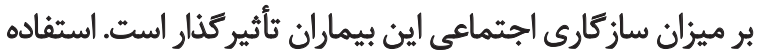

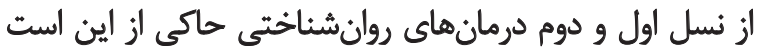

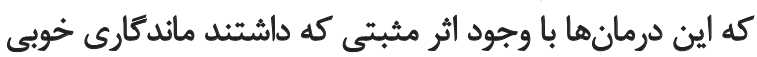

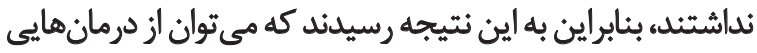

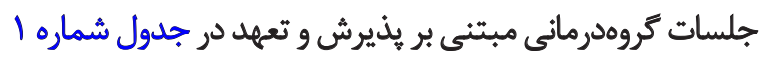
به صورت خلاصه آمده است.

ياقتهها

در جدول شماره Y ميانكين و انحراف استاندارد متغيرهاى

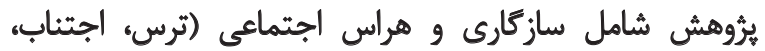

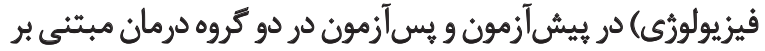

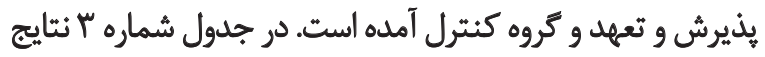

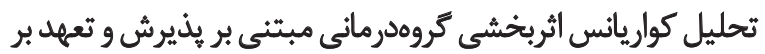

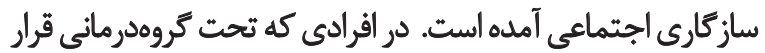

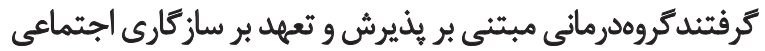

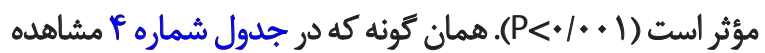

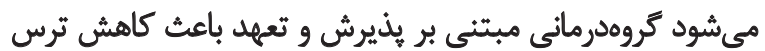

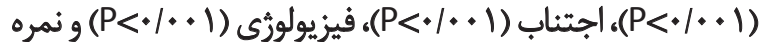

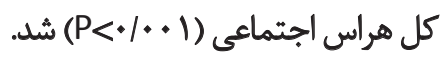
بحث

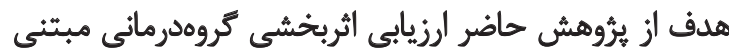

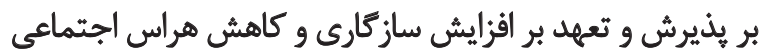

23. Mosher \& Danoff

جدول r. ميانكين (انحراف استاندارد) متغيرهاى اصلى ثرؤهش در دو كروه آزمايش و كثترل در بيش آزمون و بسآزمون

\begin{tabular}{|c|c|c|c|c|c|c|}
\hline \multicolumn{2}{|c|}{ يسأزمون } & \multicolumn{2}{|c|}{ يبيشآزمون } & \multirow{2}{*}{ كروه } & \multirow{2}{*}{\multicolumn{2}{|c|}{ مثغير }} \\
\hline انحراف معيار & مياتكين & افحراف معيار & مياتكين & & & \\
\hline $\mid H / . r$ & TA/Fq & $1 T / \pi$ & $\Delta Q / r$. & أزمايش & & سازكارى \\
\hline V/gr & $\varepsilon 9 / \pi$ & VIFe & $9 \pi / T$ & كتترل & & اجتماعى \\
\hline $8 / .9$ & $r / 4$. & $\Delta / \mathscr{C A}^{\prime} \mathrm{A}$ & IT/ & آزمايش & & \\
\hline.$/ F A$ & F/98 & $\cdot / A F$ & ir & كتترل & קرس & \\
\hline$r / \Delta V$ & $r / 9 \Psi$ & SIAS & $10 / \%$ & آزمايش & لجتئاب & \\
\hline $1 / 49$ & $1 / 48$ & $1 / 79$ & WTIT & كنترل & & هراس اجتماعي. \\
\hline $1 / P A$ & $1 / M$ & $V / A$. & $V / A$. & أزمايش & & \\
\hline $\mathrm{NAN}$ & Fise &.$/ P A$ & $V / T \pi$ & كتترل & 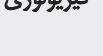 & \\
\hline$\Delta / \Delta T$ & $V 1.5$ & $\mid \Psi / . r$ & ro/fg & آزمايش & $k$ & \\
\hline r ז & $10 / \pi$ & $r / T r$ & TV/gq & كتترل & & \\
\hline
\end{tabular}

جدول ؟.نتايج تحليل كواريانس تأثير كروه درمانى مبتنى بر بذيرش و تعهد بر سازتخارى اجتماعى

\begin{tabular}{|c|c|c|c|c|c|c|}
\hline توان آزمون & اندازه اثر & Sig. & $\mathrm{F}$ & MS & منبع تغييرات & متغير \\
\hline 1 &.$/ N V$ & .1 .01 & ENTEA & $A N / a W$ & ييش أزمون & سازكاري اجتماعى \\
\hline
\end{tabular}


جدول F. نتايج تحليل كواريانس تأثير كروه درمانى مبتنى بر يذيرش و تعهد بر هراس اجتماعى

\begin{tabular}{|c|c|c|c|c|c|c|}
\hline توان أزمون & اندازه اثر & Sig. & $F$ & MS & منبع تنييرات & متغير \\
\hline $.1 .9+$ & זו//. & $+/ \Delta p q$ & A & + MAT & ييشآزمون & \multirow{2}{*}{ ترس } \\
\hline . /ave & $. / 2 \gamma / A$ & $1 / .+1$ & 1 & thus & كروه & \\
\hline.$/ I r$ & .1 .51 & . NAr & - mar & rePa & ييشأزمون & \multirow{2}{*}{ الجتناب } \\
\hline ./arp & / & $.1 . .1$ & $1 Y / 9 \mid \Delta$ & $\Delta V / / T T$ & كروه & \\
\hline.$/ 9 \Delta 1$ & TTr/T & .1 .01 & $\mid \psi / .4$ & $11 / 179$ & ييشآزمون & \multirow{2}{*}{ فيزيولوزى } \\
\hline ./49 & . NAS & $.1 . .1$ & $n / R T$ & $v \cdot / \Delta \cdot q$ & كروه & \\
\hline$\cdot \pi \cdot 9$ & $1+p q$ & . / req & $1 / r M$ & Tr/ase & يششآزمون & \multirow{2}{*}{ هراس اجتماعى كل } \\
\hline.$/ 999$ &.$|A|$. & $.1 . .1$ & $r N \cdot r$. & PAN/MT & كروه & \\
\hline
\end{tabular}

سازكًارى اجتماعى معلولان ذهنى مؤثر است [YF]. نتايج يك

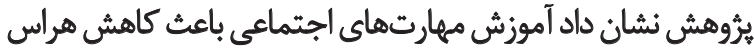

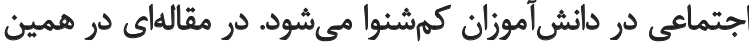

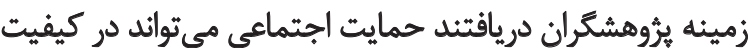

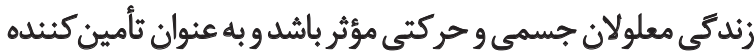

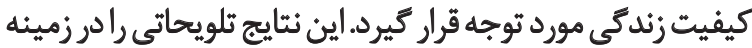

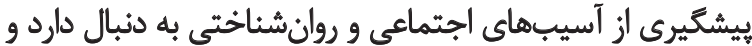

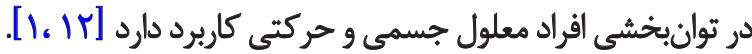

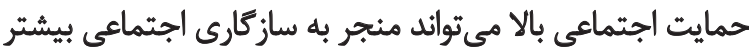
معلولان و كاهش هراس اجتماعي آنان شود.

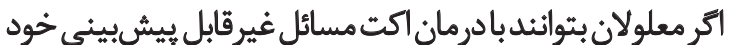

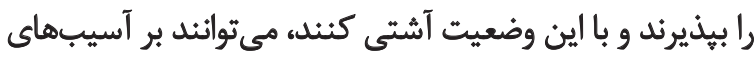

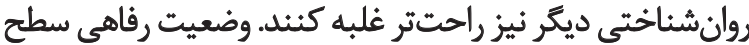

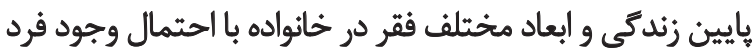

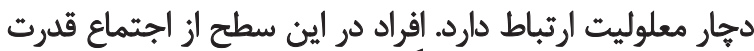

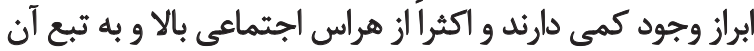

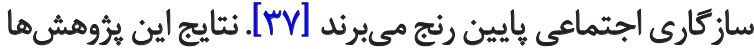

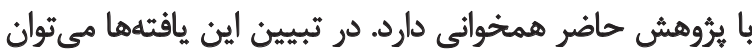

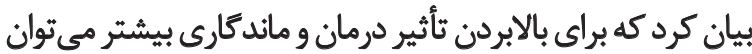

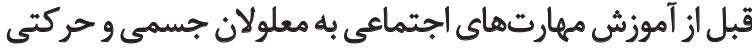

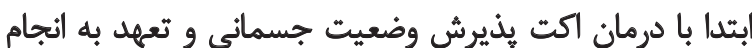

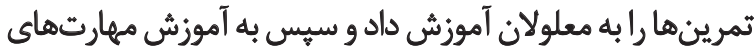

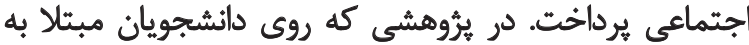

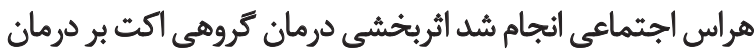

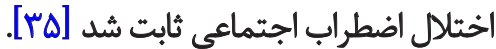

نتايج حاصل بيانكر نتايج درمان انفرادى اكت در دانشجويان

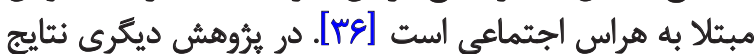

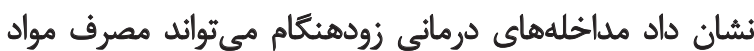

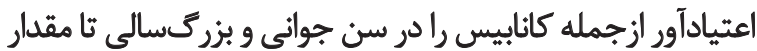

با بازدهى و ثبات بيشتر بهره برد.

امروزه با نسل سوم اين نوع درمانها مواجه هستيم كه مي توان

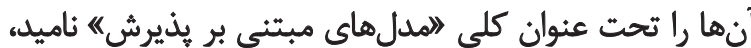

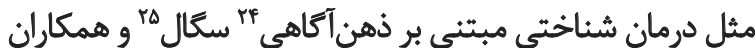

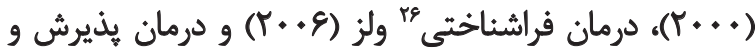

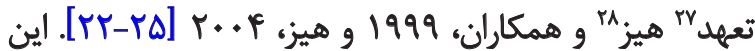

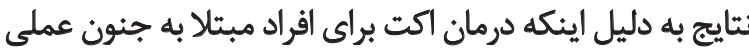

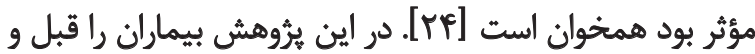

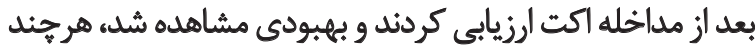

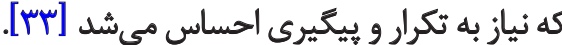
در بيروهشى ديكر اين نتيجه به دست آمد كه درمان اكت براي

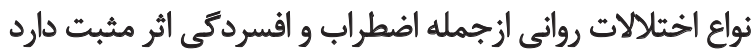

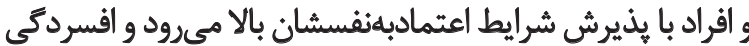

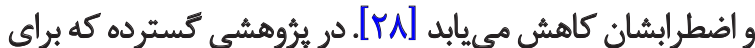

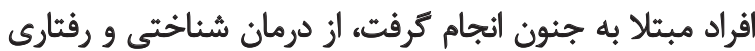

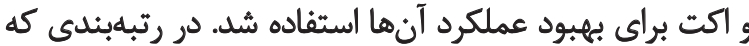

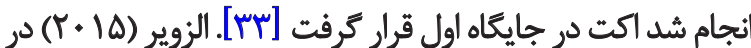

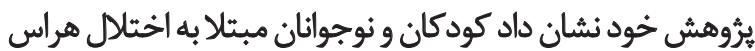

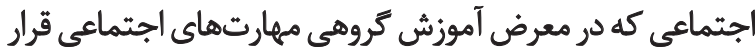

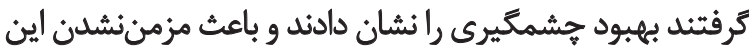

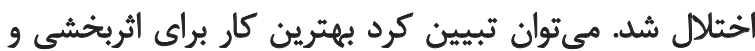

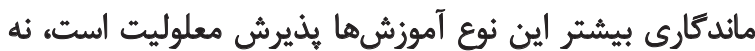

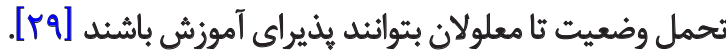
در ئروهشى ثابت شد كه آموزش مهارتهاى اجتماعى بر بهبود

24. Mindfulness

25. Segal

26. MCT

27. ACT

28. Hayes 


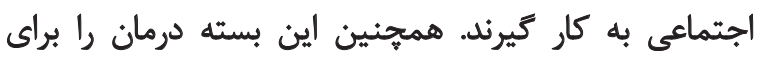

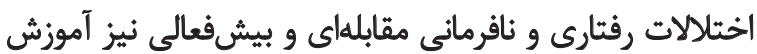

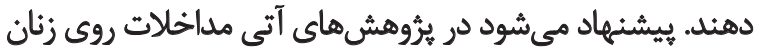

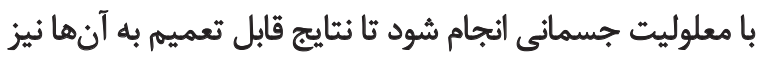

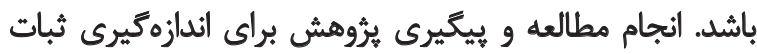

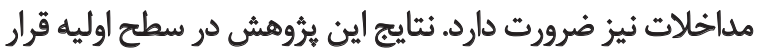

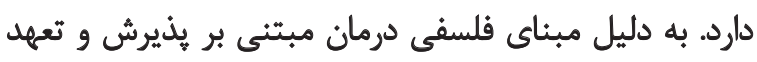

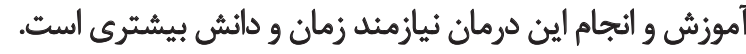

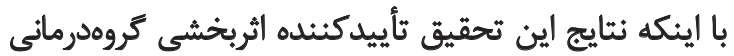

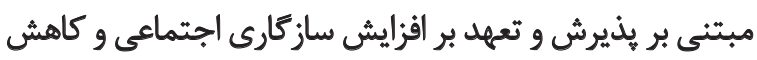

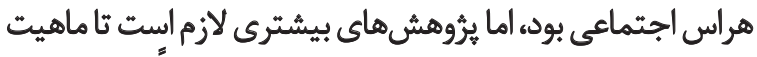

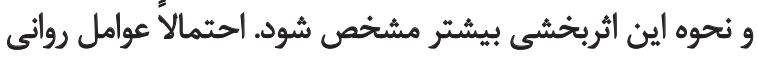

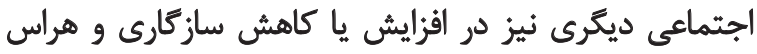

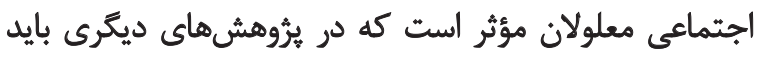

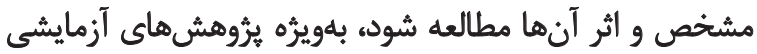

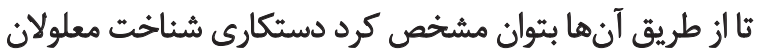

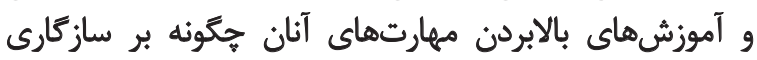

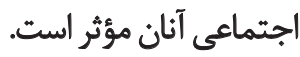

$$
\text { تشكر و قدردانى }
$$

بدين وسيله از تمامى شركت كنندكان در يرؤهش و مسئولان

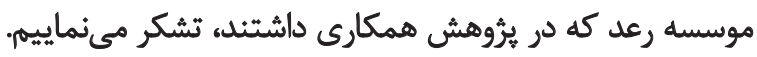
اين مقاله حامي مالى نداشُته است.
زيادى كاهش دهد و از اضطراب و افسردكى و يونيامدهاى ديكر

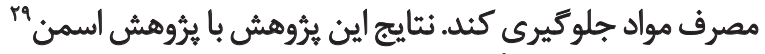

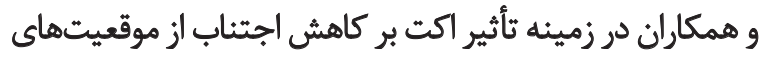

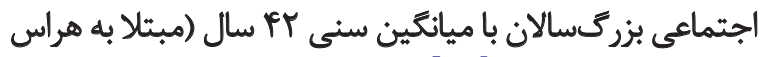

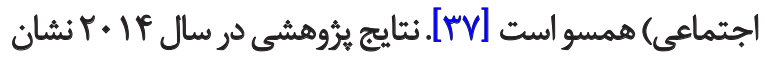

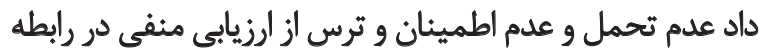

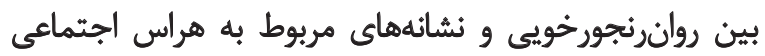

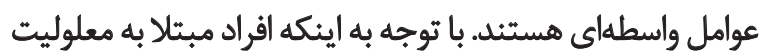

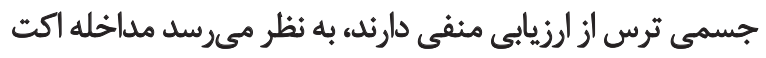

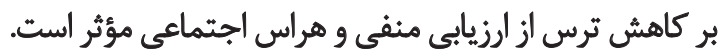

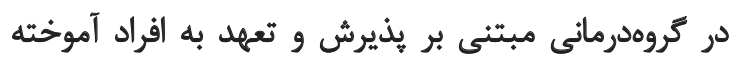

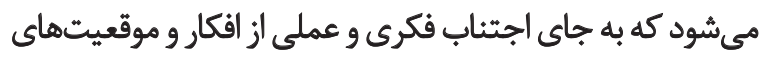

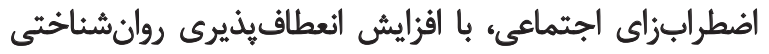

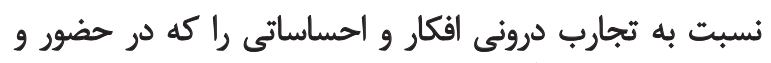

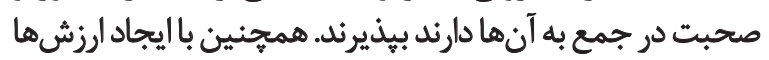

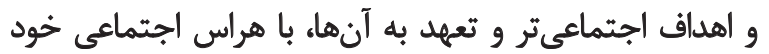

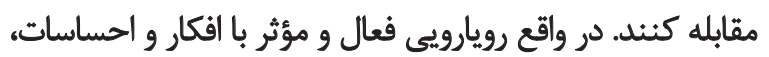

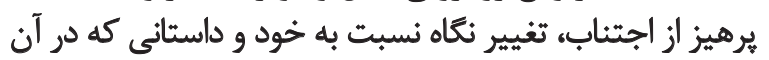

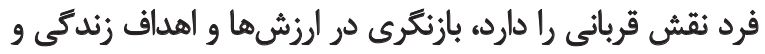

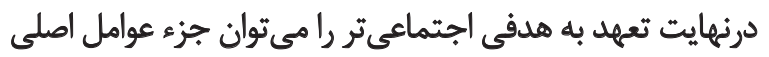

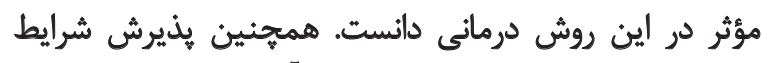

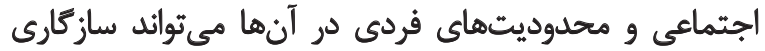

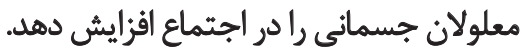

\section{نتيجليَيرى}

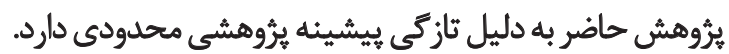

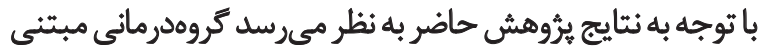

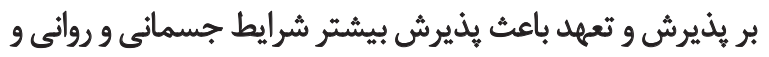

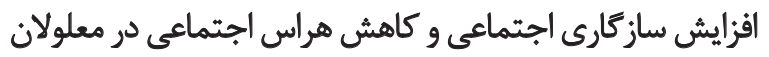

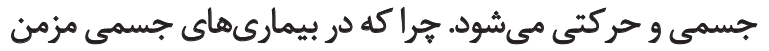

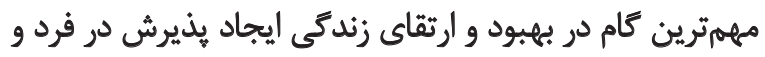

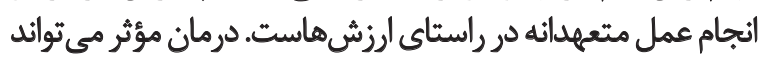
از خوددرمانى و انجام راهكارهاي نادرست جلوكيرى كند.

يكى از محدوديتهاى يروهش حاضر اين است كه نمونه

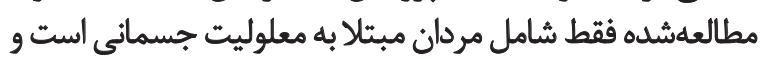

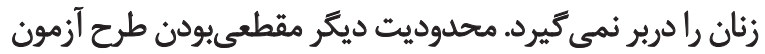

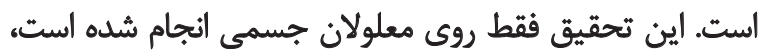

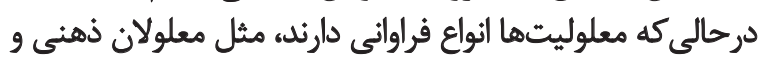

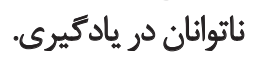

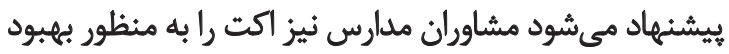

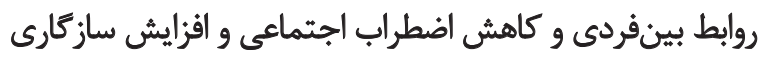




\section{References}

[1] Khosravi N, Raheb Gh, Arshia M, Eghlima M. [The relationship between social support and quality of life, physical-motor disabilities and educational center in Ra'ad (Persian)]. Rehabilitation. 2015; 16(2):176-186

[2] Barati Sede F, Davarmnesh A. [An introduction to rehabilitation of the disabled (Persian)]. Tehran: Roshd Pub; 2007.

[3] May LA, Warren S. Measuring quality of life of persons with spinal cord injury: External and structural validity. Spinal Cord. 2002; 40(7):341-50. doi: 10.1038/sj.sc.3101311

[4] Shafiabadi A. [Vocational rehabilitation for the disabled (Persian)]. Isfahan: Jungle Publication; 2003.

[5] Dalrymple KL, Galione J, Hrabosky J, Chelminski I, Young D, O'Brien E, et al. Diagnosing social anxiety disorder in the presence of obesity: implications for a proposed change in DSM5. Depression and Anxiety. 2011; 28(5):377-82. doi: 10.1002/ da.20794

[6] Ghayur Kazemmi F, Sepehri Shamlou Z, Mashhadi A, Ghanaei A, Pasalar F. [Compare the effectiveness of meta-cognitive therapy and neurofeedback to help reduce symptoms of anxiety in students Girl with social anxiety disorder (Persian)]. Journal of Cognitive Psychology. 2015; 4(1-2):19-30.

[7] Heinriches N, Hoffman SG. Information processing in social phobia: A critical review. Clinical Psychology Review. 2001; 21(5):751-70. PMID: 11434229

[8] Katzelnick DJ, Kobak KA, DeLeire T, Henk HJ, Greist JH, Davidson JRT, et al. Impact of generalized social anxiety disorder in managed care. American Journal of Psychiatry. 2001; 158(12):1999-2007. doi: 10.1176/appi.ajp.158.12.1999

[9] Clark DM, Wells A. A cognitive model of social phobia. In: Heimberg RG, Liebowitz MR, Hope DA, Schneier FR editors. Social Phobia: Diagnosis, Assessment, and Treatment. New York: Guilford Press; 1995.

[10] Heimberg RG, Brozovich FA, Rapee RM. A cognitive-behavioral model of social anxiety disorder: Update and extension. In: Hofmann SG, Dibartolo PM editors. Social Anxiety: Clinical, Developmental, and Social Perspectives. New York: Springer. 2010.

[11] Caron J, Tempier R, Mercier C, Leouffre P. Components of social support and quality of life in severely mentally ill, low income individuals and a general population group. Community Mental Health Journal. 2011; 34(5):459-75. PMID: 9793737

[12] Mosher CE, Danoff-Burg S. A review of age differences in psychological adjustment to breast cancer. Journal of Psychosocial Oncology. 2006; 23(2-3):101-14. doi: 10.1300/j077v23n02_07

[13] Rostami M, Veisi N, Jafarian Dehkordi F, Alkasir E. Social anxiety in students with learning disability: Benefits of acceptance and commitment therapy. Practice in Clinical Psychology. 2014; 2(4):277-284.

[14] Arrindell W, Sanderman R, Hageman WJJ, Pickersgill M., Kwee MG., Van der Molen H., et al. Correlates of assertiveness in normal and clinical samples: A multidimensional approach. Advances in Behaviour Research and Therapy. 1990; 12(4):153-282. doi: 10.1016/0146-6402(90)90004-a

[15] Wagner E. Development and implementation of a curriculum to develop social competence for students with visual impairments in Germany. Journal of Visual Impairment \& Blindness. 2004; 98(11):1-18.

[16] Wolffe K, Sacks SZ. The lifestyles of blind, low vision, and sighted youths: A quantitative comparison. Journal of Visual Impairment \& Blindness. 1997; 91(3):245-57.

[17] Nicholas JG, Geers AE. Personal, social, and family adjustment in school-aged children with a cochlear implant. Ear and Hearing. 2003; 24:69-81. doi: 10.1097/01.aud.0000051750.31186.7a

[18] Gresham FM, Elliott SN. The relationship between adaptive behavior and social skills: Issues in definition and assessment. Journal of Special Education. 1987; 21(1):167-81. doi: $10.1177 / 002246698702100115$

[19] Gresham FM. Social skills instruction for exceptional children. Theory Into Practice. 1982; 21(2):129-33. doi: 10.1080/00405848209542994

[20] Scholes-Balog KE, Hemphill SA, Evans-Whipp TJ, Toumbourou JW, Patton GC. Developmental trajectories of adolescent cannabis use and their relationship to young adult social and behavioural adjustment: A longitudinal study of Australian youth. Addictive Behaviors. 2016; 53:11-18. doi: 10.1016/j.addbeh.2015.09.008

[21] Van Houtem CMHH, Laine ML, Boomsma DI, Ligthart L, van Wijk AJ, De Jongh A. A review and meta-analysis of the heritability of specific phobia subtypes and corresponding fears. Journal of Anxiety Disorder. 2013; 27(4):379-88. doi: 10.1016/j. janxdis.2013.04.007

[22] Zargar Y; Besaknezhad S, Akhlaghi Jami J, Zemestani M. [The comparison of the effectiveness of group training of social skills and group cognitive-behavior therapy in reducing social anxiety among students (Persian)]. Urmia Medical Journal. 2015; 25(3):172-181.

[23] Segal ZV, Williams JMG, Teasdale JD. Mindfulness-based cognitive therapy for depression: A new approach to preventing relapse. $2^{\text {nd }}$ ed. New York: Guilford Press; 2002.

[24] Hayes SC. Acceptance and commitment therapy, relational frame theory, and the third wave of behavioral and cognitive therapies. Behavior Therapy. 2004; 35(4):639-665.

[25] Hayes SC, Strosahl KD, Wilson KG. Acceptance and commit ment therapy: An experiential approach to behavior change. $3^{\text {rd }}$ ed. New York: Guilford; 1999.

[26] Hayes SC, Gifford EV. The trouble with language: experiential avoidance, rules, and the nature of verbal events. Psychological Science. 1997; 8(3):170-3. doi: 10.1111/j.1467-9280.1997. tb00405.x

[27] Aldao A, Nolen-Hoeksema S, Schweizer S. Emotion-regulation strategies across psychopathology: A meta-analytic review. Clinical Psychology Review. 2010; 30(2):217-37. doi: 10.1016/j. cpr.2009.11.004 
[28] Omran, MP. [The effectiveness of acceptance and commitment group therapy in social phobia of students (Persian)]. Knowledge and Health Journal. 2011; 6(2):1-5.

[29] Annunziata AJ, Green JD, Marx BP. Acceptance and commitment therapy for depression and anxiety. In: Friedman $\mathrm{H}$, editor. Encyclopedia of Mental Health. Philadelphia: Springer; 2016

[30] Öst LG, Cederlund R, Reuterskiöld L. Behavioral treatment of social phobia in youth: Does parent education training improve the outcome? Behaviour Research and Therapy. 2015; 67:19-29. doi: 10.1016/j.brat.2015.02.001

[31] Connor KM, Davidson JR, ChurchillLE, Sherweed A,Foa EB. Psychometric properties of the Social Phobia Inventory (SPIN): New self-rating scale. The British Journal of Psychiatry. 2000; 176(4):379-86. doi: 10.1192/bjp.176.4.379

[32] Riahi Nia A. [The comparison of social adjustment and mental health of male and female married nurses and staff in Ahvaz city (Persian)] [MSc. thesis]. Ahvaz: Islamic Azad University, Ahvaz Branch; 2001.

[33] Zemestani M, Hasannejad L, Nejadian A. [Comparison of quality of life, sleep quality and social adjustment of cancerous patients with intact individual in Ahvaz City (Persian)]. Urmia Medical Journal. 2013; 24(7):471-482.

[34] Johns LC, Oliver JE, Khondoker M, Byrne M, Jolley S, Wykes T, et al. The feasibility and acceptability of a brief Acceptance and Commitment Therapy (ACT) group intervention for people with psychosis: The "ACT for life" study. Journal of Behavior Therapy and Experimental Psychiatry. 2016; 50:257-63. doi: 10.1016/j. jbtep.2015.10.001

[35] Beh Pajooh A, Soleymani S, Afrooz GA, Gholam Ali Lavasani M. [A study on the impact of social skill training on social adjustment and academic performance in slow learners (Persian)]. Quarterly Journal of Educational Innovations. 2011; 33(9):163- 186.

[36] Pourfaraj Omran M. [The effectiveness of acceptance and commitment group therapy in social phobia of students (Persian)]. Journal of knowledge and Health. 2012; 6(2):1-5. doi: 10.1234/ knh.v6i2.100.g61

[37] Block JA, Wulfert E. Acceptance or change: Treating socially anxious college students with ACT or CBGT. The Behavior Analyst Today. 2000; 1(2):3-10. doi: 10.1037/h0099879

[38] Ossman WA, Wilson KG, Storaasli RD, McNeill JW. A preliminary investigation of the use of acceptance and commitment therapy in group treatment for social phobia. International Journal of Psychology \& Psychological Therapy. 2006; 6(3):397-416. 